\title{
Tangence
}

\section{L'être en suspens}

\section{Jacques La Mothe}

Numéro 38, décembre 1992

Fiction policière et roman actuel

URI : https://id.erudit.org/iderudit/025739ar

DOI : https://doi.org/10.7202/025739ar

Aller au sommaire du numéro

Éditeur(s)

Tangence

ISSN

1189-4563 (imprimé)

1710-0305 (numérique)

Découvrir la revue

Citer cet article

La Mothe, J. (1992). L'être en suspens. Tangence, (38), 65-73.

https://doi.org/10.7202/025739ar d'utilisation que vous pouvez consulter en ligne.

https://apropos.erudit.org/fr/usagers/politique-dutilisation/ 


\section{L'être en suspens}

\section{Jacques La Mothe}

\section{* L'artiste est un suspect * 1}

Lorsque la voix narrative du Double assassinat dans la rue Morgue, que Jules Verne assimile immédiatement à Edgar Poe ${ }^{2}$, nous dévoile le portrait d'Auguste Dupin, c'est par le biais des livres, de l'écriture et de la ville de Paris identifiée comme la ville des livres:

Les livres étaient véritablement son seul luxe, et à Paris, on se les procure facilement. ${ }^{3}$

Je fus étonné de la prodigieuse étendue de ses lectures.

Dans La lettre volée, Dupin ira jusqu'à s'avouer "coupable d'une certaine rapsodie ": doggerel signifiant en fait vers de mirliton...

C'est aussi par le biais des livres et de l'écriture que nous obtenons des renseignements sur la conjonction qui naît entre Dupin et cette voix narrative:

Notre première rencontre se fit dans un obscur cabinet de lecture de la rue Montmartre, par ce fait fortuit que nous étions tous deux à la recherche d'un même livre fort remarquable et fort rare; cette coïncidence nous rapprocha. Nous nous vîmes toujours de plus en plus.

Cherchant dans Paris certains objets qui faisaient mon unique étude, je vis que la société d'un pareil homme serait pour moi un trésor inappréciable, et dès lors je me livrai franchement à lui. Nous décidâmes enfin que nous vivrions ensemble tout le temps de mon séjour dans cette ville.

1 Jean-Paul Sartre, *Masson *, Situations $I V$, Paris, Gallimard, 1964, p. 387. La phrase complète se lit comme suit: "L'artiste est un suspect; n'importe qui peut le questionner, l'arrêter et le traîner devant les juges; toutes ses paroles, toutes ses œuvres peuvent être retenues contre lui.*

2 Jules Verne, adgar Poe et ses cuvres „, Le musée des familles, avril 1864, 31' volume, p. 193.

3 Edgar Allan Poe, Contes. Essais. Poèmes, Paris, Robert Laffont, 1989. Toutes les citations de Poe sont tirées de ce livre. 
66

"Nous ne vivions qu'entre nous", ajoute la voix narrative et si la bizarrerie d'humeur de son ami "c'était d'aimer la nuit pour l'amour de la nuit ", elle aussi tombe dans cette bizarrerie jusqu'à contribuer à en faire la contrefaçon:

Au premier point du jour, nous fermions tous les lourds volets de notre masure, nous allumions une couple de bougies fortement parfumées, qui ne jetaient que des rayons très faibles et très pâles. Au sein de cette débile clarté, nous livrions chacun notre âme à ses rêves, nous lisions, nous écrivions, ou nous causions, jusqu'à ce que la pendule nous avertît du retour de la véritable obscurité."

Scansion des douze coups qui modulent la contrefaçon de la nuit:

Alors nous nous échappions à travers les rues, bras dessus bras dessous, continuant la conversation du jour, rôdant au hasard jusqu'à une heure très avancée, et cherchant à travers les lumières désordonnées et les ténèbres de la populeuse cité ces innombrables excitations de l'esprit que l'étude paisible ne peut pas donner.

Oscillation entre un Dupin créateur et un Dupin analyste, âme double que Poe illustre par cette anecdote dans laquelle Dupin retrace la rêverie de la personne qui l'accompagne, âme double qui chez Baudelaire va se multiplier dans cet art de jouir de la foule:

Le poète jouit de cet incomparable privilège qu'il peut à sa guise être lui-même et autrui, comme ces âmes errantes qui cherchent un corps, il entre, quand il veut, dans le personnage de chacun. Pour lui seul, tout est vacant; et si certaines places paraissent lui être fermées, c'est qu'à ses yeux elles ne valent pas la peine d'être visitées. ${ }^{4}$

Dans le récit de Poe, qui s'enracine à la fois dans "La genèse d'un poème * et dans "Eurêka ", le livre, la lecture et l'écriture vont apparaître non comme une métaphore, mais comme un procédé formel qui joue le rôle d'écho au questionnement lié à l'élaboration d'une ceuvre et à la part artistique de l'individu; si "The universe is the plot of God " (L'univers est l'intrigue de Dieu "), le livre en est le modèle réduit.

4 Charles Baudelaire, "Les foules. (Petits poèmes en proses), Euvres complètes, Paris, Robert Laffont, coll. *Bouquins», 1980, p. 170. 
Plutôt que de doter le monde d'une méthode policière comme le croyait Breton, qui en passant crachait sur Edgar Poe ${ }^{5}$, le récit de Poe apparaît comme un texte à multiples registres, discursif par la problématique qui y est énoncée, lyrique par les intrigues narratives qui illustrent ces observations, encyclopédique par son ouverture qui constitue une invite au lecteur non pas à déconstruire l'énigme apparente (puisque c'est par Dupin qu'une solution inédite est trouvée), mais plutôt à pénétrer par la pratique dans le laboratoire de l'imagination. Ce faisant, le secret du texte se révèle progressivement grâce à l'intercession du lecteur transformé en écrivain potentiel. On voit dès lors que l'essentiel va porter sur le langage et la littérature.

\section{Registre discursif}

Double assassinat dans la me Morgue s'ouvre sur une série -d'observations jetées tout à fait à l'abandon", propositions portant sur la difficulté d'analyser *les facultés de l'esprit qu'on définit par le terme "analytique" "que nous n'apprécions que par leurs résultats. Cette faculté de résolution joue sur les mots, puisqu'elle est assimilée aux mathématiques, à la résolution d'une énigme, au travail de l'analyse, dans la mesure où elle permet de décomposer la pensée en ses éléments, de combiner ces éléments de façon à permettre de dévoiler ce qui ne veut pas se laisser dire de prime abord, enfin, c'est aussi l'état d'esprit d'une personne qui prend une détermination, une décision volontaire et qui s'y tient.

Les résultats de cette faculté de *résolution " se laissent percevoir faussement comme une intuition alors que le processus de résolution est lié à une qualité de l'observation: "le principal est de savoir ce qu'il faut observer."

Comparant le jeu d'échecs, le whist et le jeu de dames, ce dernier étant privilégié puisqu'au contraire des deux autres jeux le mouvement y est "simple dans son espèce et ne subit que peu

5 André Breton, second manifeste du surréalisme Manifestes du surréalisme, Paris, Gallimard, coll. ، Idées ", 1930, p. 81. Ia phrase complète se lit comme suit: *N'est-ce pas une honte de présenter sous un jour intellectuellement séduisant un type de policier, toujours de policier, de doter le monde d'une méthode policière? Crachons, en passant, sur Edgar Poe. * 
de variations, les probabilités d'inadvertance sont beaucoup moindres, et l'attention n'étant pas absolument et entièrement accaparée, tous les avantages remportés par chacun des joueurs ne peuvent être remportés que par une perspicacité supérieure ", le texte met en opposition l'ingéniosité et l'aptitude analytique, la songerie et l'imagination, Dupin étant homme d'imagination, donc d'analyse.

Ainsi, à un jeu de dames dont la totalité des pièces serait réduite à quatre pièces, la victoire ne pourrait être décidée que par une tactique recherchée, "résultat de quelque puissant effort de l'intellect. . Ce carré de dames, le texte le fournit: quatre éléments auxquels répondra un autre carré de personnages dans le singulier récit qui suit cette préface.

\section{Registre lyrique}

Le suspense qui sous-tend le texte à énigme provient du jeu entre la fable et l'histoire: il faut les lire ici dans leurs rapports avec le langage.

Le récit du crime raconte la capture, dans l'archipel indien, d'un orang-outang (du malais: homme des bois) par deux matelots dont l'un mourra par la suite. L'homme des mers loge le singe captif dans sa demeure parisienne. Le singe s'enfuit. Vient ensuite le récit de la poursuite et la description du meurtre d'une veuve et de sa fille dont le patronyme, L'Espanaye, peut suggérer *explanation. Les corps sont retrouvés l'un noir de suie, l'autre baignant dans son sang.

Le premier carré, fort suggestif pour une étude à caractère mythocritique, est maintenant réduit à deux: les dames éteintes, restent les * damned ", tandis qu'on retranche un des douze témoins, incarcéré •bien que rien dans les faits déjà connus ne parût suffisant pour l'incriminer *

Dupin et la voix narrative, exclus de la fable, se retrouvent dans l'histoire, où il est question de la façon dont on découvre que le meurtrier ne peut être qu'un orang-outang et de la manière dont Dupin entre en contact avec son propriétaire. On lit donc la recension dans la Gazette des douze récits partiels de la fable. Chaque témoin en interprète un segment limité par son aire encyclopédique et, tandis que Dupin prend connaissance de cette fable, soit par des témoignages, soit de façon directe, la recons- 
tituant, la dévoilant, la voix narrative, spectatrice de cette reconstitution, nous retransmet son élaboration, son invention.

À ce jeu entre le récit de la fable, sa lecture et la narration qui en est faite, une dimension temporelle prend corps puisque l'intrigue doit être comprise progressivement; mais le texte de Poe recèle aussi un «temps évolutif ", plus subtil que «le temps de lecture " dont il parle dans Genèse d'un poème, puisqu'il s'agit d'un temps ouvert, sans terme ni conclusion prévisibles, mais qui implique les lecteurs comme intervenants dans cette épaisseur nouvelle du texte déjà complexe par sa trame de niveaux narratifs d'ordres divers.

\section{Registre encyclopédique}

Puisque le récit d'énigme est aussi un jeu, on peut s'amuser à en résumer chaque séquence en une phrase. Nous aurons ainsi:

- quelques observations sur la faculté de résolution;

- récit de la conjonction entre la voix narrative et Auguste Dupin;

- exemple de rêverie interprétée par Dupin;

- relation générale d'un double crime dans une première édition de la Gazette des tribunaux;

- relation des douze témoignages dans le numéro suivant;

- dans l'édition du soir: arrestation de l'un des témoins;

- intérêt de Dupin pour l'affaire et visite des lieux;

- déductions de Dupin concernant les voix entendues;

- déductions de Dupin concernant le meurtrier et son complice involontaire;

- convocation et témoignage du propriétaire de l'orangoutang;

- réflexion conclusive de Dupin.

Il manquera évidemment la première de ces séquences, celle que constitue la trop évidente épigraphe de Sir Thomas Browne, qu'un regard oblique nous fait découvrir, présidant au dialogue des textes: 
70

Quelle chanson chantaient les Sirènes? Quel nom Achille avait-il pris, quand il se cachait parmi les femmes? - questions embarrassantes, il est vrai, mais qui ne sont pas situées au-delà de toute conjecture.

On ne peut s'empêcher de relier cela à la dernière phrase du récit, non celle prononcée par Dupin mais celle que Poe prélève dans La nouvelle Hélotse:

Tout, dans un poème comme dans un roman, dans un sonnet comme dans une nouvelle, doit concourir au dénouement. Un bon auteur a déjà sa dernière ligne en tête quand il écrit la première. (La Genèse d'un poème)

Ainsi:

Je veux parler de sa manie «de nier ce qui est, et d'expliquer ce qui n'est pas !

1. Rousseau, Nouvelle Héloüse. - E.A.P.

Mais pourquoi ces allusions interrogatives à des mythes anciens? Pourquoi ce prélèvement d'un texte plus récent, cet encadrement auquel le récit d'énigme sert de mise en abyme?

Les Sirènes étaient des femmes-oiseaux, des oiseaux à tête de femme et aux caractères féminins très marqués, avant de devenir des femmes-poissons pour avoir perdu leurs ailes à la suite d'un concours de chant avec les Muses. Robert Graves ${ }^{6}$ en répertorie onze:

Aglaopé - beau visage

Aglaophonos - belle voix

Leucosia - créature blanche

Ligéia - cri perçant

qui nous renvoient au conte favori de Poe,

Molpé - musique

Parthénopé - visage de jeune fille

Persinoë - persuasive

Raidné - progrès

Télès - parfaite

6 Les informations relatives à la mythologie grecque sont tirées de Robert Graves, Les mythes grecs, Paris, Fayard, Paris, coll. «Pluriel *, 1967, et de Géza Róheim, Les portes du rêve, Paris, Payot, coll. *Science et l'homme", 1973. 
Thelxépéïa - paroles apaisantes

Thelxiope - visage convaincant,

mais elles ne sont jamais toutes les onze ensemble comme les Muses sont neuf; elles sont perçues en leur vol tourbillonnaire et Circé met en garde Ulysse contre leurs maléfices: selon l'étymologie ce sont "celles qui étouffent *.

Si le douzième chant de L'odyssée nous apprend que celui qui entend leurs voix enchanteresses s'en va content et plus riche en savoir - car les Sirènes savent tous les maux que les dieux ont infligés aux gens d'Argos et de Troie, ainsi que tout ce que voit passer la terre nourricière -, il est extrêmement difficile de répondre à la question posée puisque leur chant remplissait le cœur du désir d'écouter, à un point tel que les matelots, charmés, perdaient la raison et en mourraient: nul témoin n'a entendu leur chant en son entier. Ce chant est leurre du passé, le futur n'étant envisagé que sous l'aspect d'une répétition de ce passé.

Il est intéressant de rapprocher cette brève description des Sirènes de la scène des anges dans le second Faust, au moment de la mort de Faust, quand Méphistophélès parle:

Vous vous appelez les réprouvés

Et c'est vous qui êtes les vrais maîtres sorciers

Car vous séduisez l'homme comme la femme.

Oh, la maudite aventure!

Est-ce là l'élément d'amour?

Par ailleurs, le fait de voir Achille au milieu des Sirènes, rapproché d'elles par cette seconde question, peut de prime abord nous étonner, mais Achille (sans lèvres) est un héros oraculaire: sa parole n'est pas étrangère au chant des Sirènes, mais d'un registre différent et, ici, complémentaire, car à l'origine affublé de vêtements féminins, il s'affirme comme héros viril, à Skyros, parmi les filles de Lycomède. Il porte alors le nom de Cercyséra (celui qui manie la quenouille); il la manie si bien que, non content de faire des travaux ménagers, il aura une aventure avec Deidamie, l'une des filles de Lycomède. Plus tard, de mauvaises langues prétendront qu'il s'éprendra de Troilos, mais que, frustré par son refus, il le tuera en lui tranchant la tête ou, selon d'autres récits, en l'étranglant.

Si l'on ajoute à cela qu'Achille, caché parmi les femmes, prit les armes au bout de quatre mois, sortant ainsi de la tutelle de sa mère, la Nuit, et que Pélée fait de Phoenix qui en recouvre la vue 
un second père pour Achille, il devient révélateur de mettre en relations certains éléments de la biographie de Poe avec ce récit mythique et certains éléments de ce récit avec des motifs du texte de Poe.

Enfin, ce polymorphisme de la sexualité des Sirènes et d'Achille a une parenté certaine avec l'incertitude que l'on retrouve à ce propos entre Dupin et la personne qui l'accompagne, tous deux tels les enfants crétois, enfants de la nuit. Il y a donc à l'origine de la création artistique un certain nombre de liens entre le polymorphisme de la sexualité adolescente - c'està-dire de la personne qui s'affranchit du troisième sexe, de l'état neutre lié à l'enfance selon Fourier - et la vie sexuelle de l'artiste, d'un point de vue métaphorique évidemment, en relation avec l'œuvre qu'il édifie.

Ia citation de La nouvelle Hélot'se indiquerait que la relation entre Dupin et la personne qui l'accompagne, cette voix narrative, est véritablement harmonieuse puisqu'à la fin de ce roman par lettres:

C'est Julie qui est le précepteur du précepteur. Saint-Preux, dans les premières pages précepteur imprudent, deviendra à la fin précepteur raisonnable des enfants de son ancienne élève et amante. ${ }^{7}$

Nous avons donc, superposée aux onze séquences du texte de Poe, auxquelles on ajoute l'épigraphe, la ronde des onze témoins dont le récit, incluant celui du banquier, ne forme plus qu'un, qui est réponse au chant des onze Sirènes, déjà en relation avec le personnage ambigu d'Achille, comme si ce texte était l'américanisation, l'actualisation d'un texte bien antérieur, mais avec cependant certaines modifications.

Par ailleurs, nous avons un premier carré de personnages: Mme de l'Espanaye et sa fille, le marin et son compagnon; le compagnon meurt, remplacé par l'orang-outang. Les deux femmes tuées, restent le marin et son singe, d'une part, et d'autre part, Dupin et sa voix accompagnatrice: l'orang-outang au Jardin des Plantes et les différentes facettes de Dupin réconciliées, "ce qui est n'étant plus nié". Sur le damier du texte demeurent l'auteur, créateur et analyste, interprète et inventeur, et le lecteur, écrivain

7 Michel Butor, aLîle du bout du monde ", Répertoire III, Paris, Minuit, Paris, 1968, p. 69. 
en suspens, détourné pour toujours de l'énigme, âmes doubles: ainsi voici reconstitué le carré d'origine, mais de bien différente façon!

\section{Envoi}

Subsistent fort heureusement quelques questions: quel est ce livre fort remarquable et fort rare dont il est question au début du texte, d'autant plus introuvable qu'il se pourrait qu'il soit encore à écrire?

Quelle est cette unique recherche, si ce n'est de trouver réponse à la question inaugurale de Nadja "Qui suis-je? Si par exception je m'en rapportais à un adage: en effet pourquoi tout ne reviendrait-il pas à savoir qui je "hante" ?.8

Quelle est cette citation latine qui clôt l'analyse de la rêverie par Dupin: :Perdidit antiquum littera prima sonum", que l'on peut traduire par *La première lettre a perdu le son ancien ", lettre non pas volée, mais en attente, en souffrance, la serpentine $S$ qui féminise le be de la langue anglaise?

Double assassinat dans la rue Morgue est certes un texte fondateur: n'y retrouve-t-on pas, en germe, le récit palimpseste et pluriel, certains éléments de l'autobiographie à caractère fictionnel, ou encore l'inclusion de la personne qui lit comme intervenant actif dans la continuité de l'écriture?

L'interprétation, restaurant le sens, se joue ici comme exercice du soupçon. Mais le vrai sens d'un texte existe-t-il ?

8 André Breton, Nadja, Paris, Le Livre de poche, 1964, p. 9. 